\title{
STUDIES ON HORMONAL TREATMENT FOR MASTOPATHY, PARTICULARLY ITS DOSE-RESPONSE RELATIONSHIP
}

\author{
MASARU IZUO AND MASAO FUJIMORI \\ Department of Surgery, Gunma University \\ School of Medicine, Maebashi
}

\begin{abstract}
SYNOPSIS
The present studies were undertaken to ascertain a rational program of hormone administration for patients with mastopathy, in view of the fact that the dosage, mode, and duration of administration have varied widely in practice. The drug employed in the present studies was 2-alpha-methyl-dihydrotestosterone propionate (dromostanolone propionate). The drug recipients were divided into four groups according to dosage level: Group M-1 received 12.5 mg, group M-2, $25 \mathrm{mg}$; group M-3, $50 \mathrm{mg}$; and group M-4, $100 \mathrm{mg}$; once per week. A control group (M-5) received $45 \mathrm{mg}$ of a depot-type testosterone once per week. The patients were assigned randomly to the groups by use of a table of random numbers. The drug was administered in double-blind fashion. The treatment produced no significantly different results with regard to spontaneous pain, tenderness, and softening of the tumor. However, regarding size the tumor diminution was proportionate to the level of dose. A similar tendency was observed in weight gain. Stratified analysis showed the tumor diminishing effect higher in the younger age group, in tumors larger in size, and in tumors poorly delineated. No age difference in the drug effect was observed in regard to weight gain. A rational administration of the present drug in patients with mastopathy seems to consist of the following: administration of $100 \mathrm{mg}$ once a week for 4 weeks; if the subsequent response is sufficient, treatment is halted, if the response is insufficient, the drug is continued for several weeks more in a reduced dose. If no response is elicited surgical managements are suggested.
\end{abstract}

A general principle concerning treatment of mastopathy has not yet been well accepted. As to the significance of hormonal treatment for mastopathy various opinions have been expressed. The authors believe that some cases of mastopathy considered to develop because of sex hormonal imbalance can be treated by means of correction in sex hormonal status.

Sex hormones used in the management of mastopathy are mainly androgens or their derivatives. Dosage, schedule of administration and duration of treatment differ in the various reports. Evaluation of therapeutic effects and side effects has not been defined distinctly until recently.

The present study is an attempt to evaluate the effectiveness and the side effects of 2alpha-methyl-dihidrotestosterone propionate, based on adequate objective criteria, and to determine a rational program of treatment for patients with mastopathy.

\section{MATERIALS AND METHODS}

In order to analyze the dose-response relationship, effectiveness and side effects of the drug

Received for publication November 7, 1966. 
Test Agent

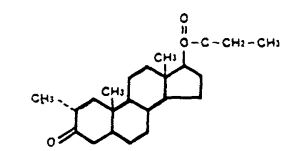

Chemical Name : $2 \alpha \cdot$ methyl-dihydrotestosterone propionate

Generic Name: Dromostanolone propionate
Control Agent

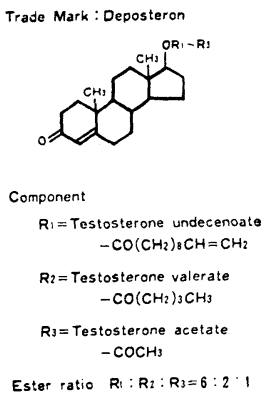

Fig. 1. Administered agents.

were evaluated on the basis of objective data. Patients were classified into 4 groups according to dosage of 2-alpha-methyl-dihidrotestosterone propionate (dromostanolone propionate), plus one group which served as a control. The drug was administered once per week intramuscularly in the following dosages: group M-1 (12.5 mg), group M-2 (25 mg), group M-3 (50 mg) and group M-4 (100 mg). A control group, M-5, received $45 \mathrm{mg}$ of depot-type testosterone intramuscularly (Fig. 1). Each group consisted of 7 or 8 cases, and randomization was achieved by use of a table of random numbers. Objectivity was maintained by use of the double-blind technique of drug administration.

Objective evidence of tumor regression was accepted as response to therapy. The boundary of the tumor was determined by palpation, and on this basis the involved area was measured by means of a planimeter. Other clinical observations included spontaneous pain, tenderness, softening of the tumor, body weight, menstrual disorders, basal body temperature, hoarseness, edema, acne and hirsutism.

Stratification of patients was restricted to 3 variants, age of patients ( 2 categories), size of the tumor ( 2 categories) and border of the tumor ( 2 categories).

\section{RESULTS}

Of 40 patients admitted to this study 2 were excluded because of insufficient observation, therefore 38 patients were evaluated.

Figure 2 shows effect of the drug on spon-

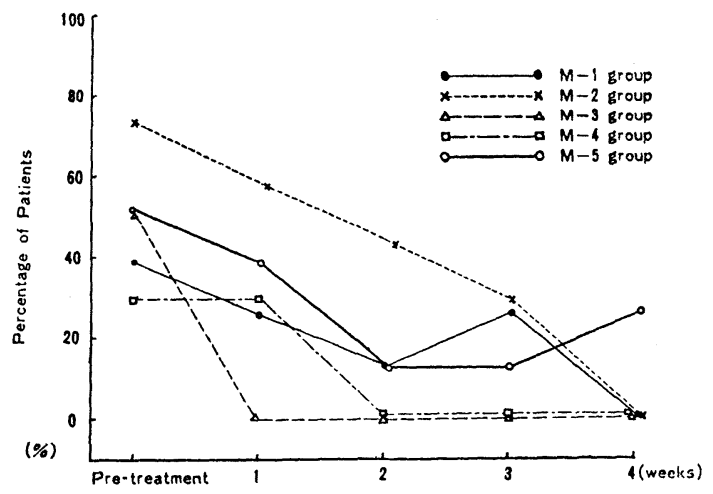

Fig. 2. Percentage of patients with spontaneous pain.

taneous pain during each week. In the third week pain remained in about $30 \%$ of groups M-1 and M-2. In the fourth week pain was absent in all patients except group M-5, in which the drug took effect somewhat later. As to effect on tenderness to pressure, a similar tendency was otserved, with apparently no particular difference among the groups.

Softening of the tumor was found in different weeks. As seen in Figure 3, softening effects were observed at a rate of $90 \%$ to $100 \%$ in groups $\mathrm{M}-1$ through $\mathrm{M}-4$ in 3 to 4 weeks, while the response in control M-5 was lower.

Response of tumor size is shown in Figure 4 with regression lines representing the average variations in each week. Significant linear regression was observed in groups $\mathrm{M}-1, \mathrm{M}-3$ and M-4. A survey of relative significance among the 5 groups revealed significant differences in groups $M-1, M-3$ and M-4. An

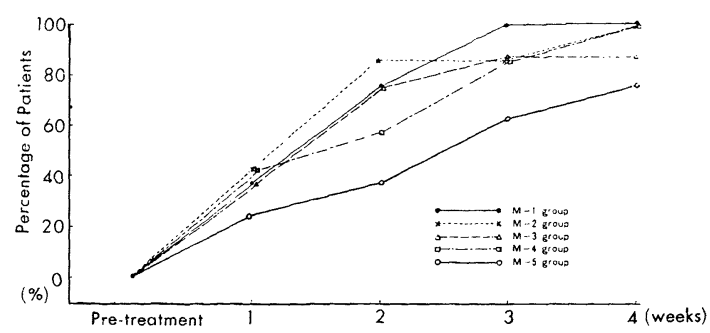

Fig. 3. Percentage of patients with softening of tumor. 
Table 1. Distribution of patients with side effects (post-treatment)

\begin{tabular}{ccccccc}
\hline Group & $\begin{array}{c}\text { No. of } \\
\text { patients }\end{array}$ & $\begin{array}{c}\text { Menstrual } \\
\text { Disorders }\end{array}$ & Hoarseness & Hirsutism & Edema & Acne \\
\hline M-1 & 8 & 0 & 0 & 0 & 0 & 1 \\
M-2 & 7 & 1 & 0 & 0 & 0 & 1 \\
M-3 & 8 & 0 & 0 & 0 & 0 & 0 \\
M-4 & 7 & 1 & 0 & 1 & 0 & 1 \\
M-5 & 8 & 1 & 1 & 1 & 0 \\
\hline
\end{tabular}

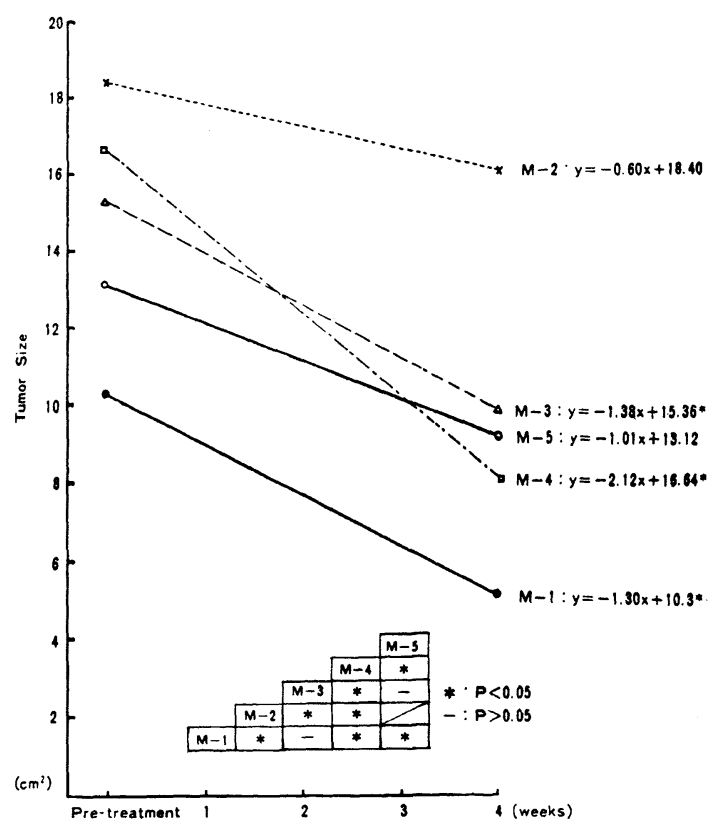

Fig. 4. Linear regression of tumor size.

interesting finding in this connection was that in group M-3 and M-4 the drug effect was more evident than in control group M-5.

Stratified analysis between certain factors and the response on tumor size was subsequently undertaken. Age difference was first examined, each group being divided at 40 years of age. As presented in the left graph of Figure 5, the drug effect was higher in the younger section of groups $\mathrm{M}-1$ to $\mathrm{M}-4$. But this tendency of higher effectiveness in the younger patients was not distinct in only group M-4 showing better clinical results, as presented in the right graph of Figure 5.

Groups were then divided into two categories by tumor size: larger tumor $\left(15 \mathrm{~cm}^{2}\right.$ or larger) and smaller tumor (14 $\mathrm{cm}^{2}$ or less). The larger tumor group responded better to treatment in groups M-1 through M-4 and also in group M-4 alone (Fig. 6).

As shown in Figure 7, dividing the cases into two groups regarding tumor border, those with tumors of indefinite border showed better response to the drug in all groups of M-1 to M-4 (left graph in Fig. 7). In group M-4 alone, however, there was no significant difference (right graph in Fig. 7).

Body weight gain, which is considered a side effect in treatment of mastopathy, was then studied in terms of its variations and converted into regression lines. A correlation between the dose and weight gain was observed in all groups, particularly with significant difference in groups M-3 and M-4 (Fig. 8). In stratified linear analysis of age on weight gain, no particular difference was found between older and younger patients (Fig. 9), differing from the response on tumor size.

Incidence of other side effects is exhibited in Table 1. No consistent tendency was observed. Effect of the drug on the menstrual cycle was examined on the basis of basal body temperature. According to these data, there was no distinct effect in groups $\mathrm{M}-1, \mathrm{M}-2$ and $M-3$, while in group $M-4$, which received the highest dose of drug, one case shifted from a normal to a deranged cycle. One climacteric case in control group M-5 exhibited a functional bleeding-like change, presumed to be the effect of the drug.

\section{DISCUSSION}

The empirical use of estrogens was prac- 

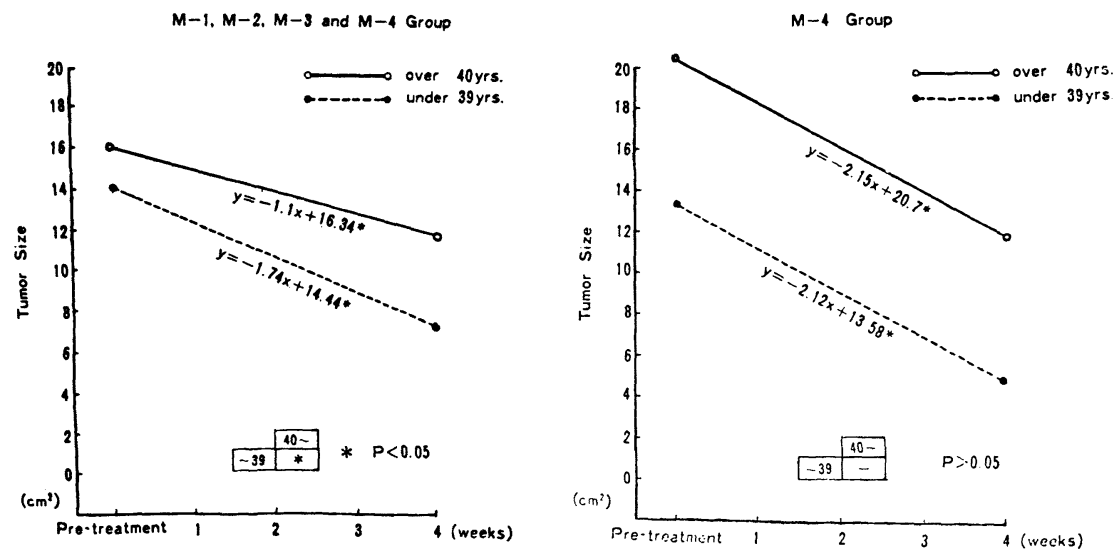

Fig. 5. Linear regression of tumor size, stratified according to age.
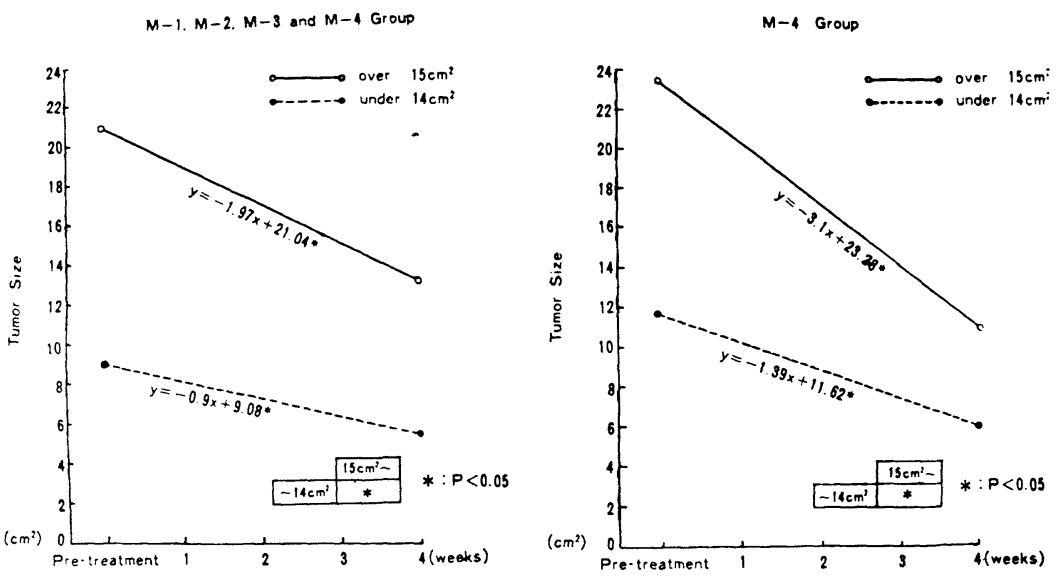

Fig. 6. Linear regression of tumor size, stratified according to tumor size.
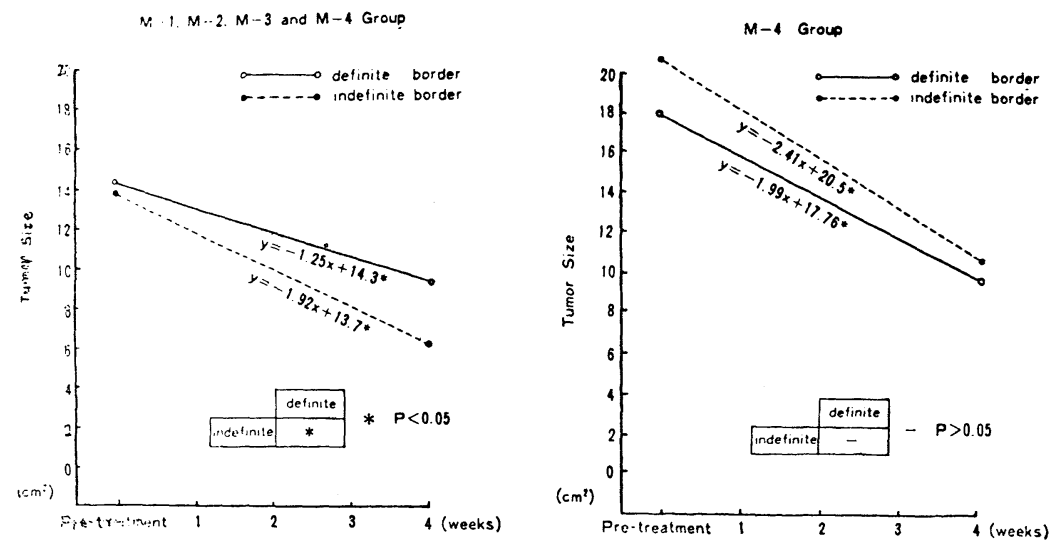

Fig. 7. Linear regression of tumor size, stratified according to tumor border. 
tised more than 30 years ago in hormonal treatment for mastopathy, when the sex hormonal imbalance constituting the background of this disease had not been sufficiently clarified. Since learning that mastpathy is ascribable to relative hyperestrogenism, treatment with antagonizing hormones has come into use, such as corpus luteum hormone

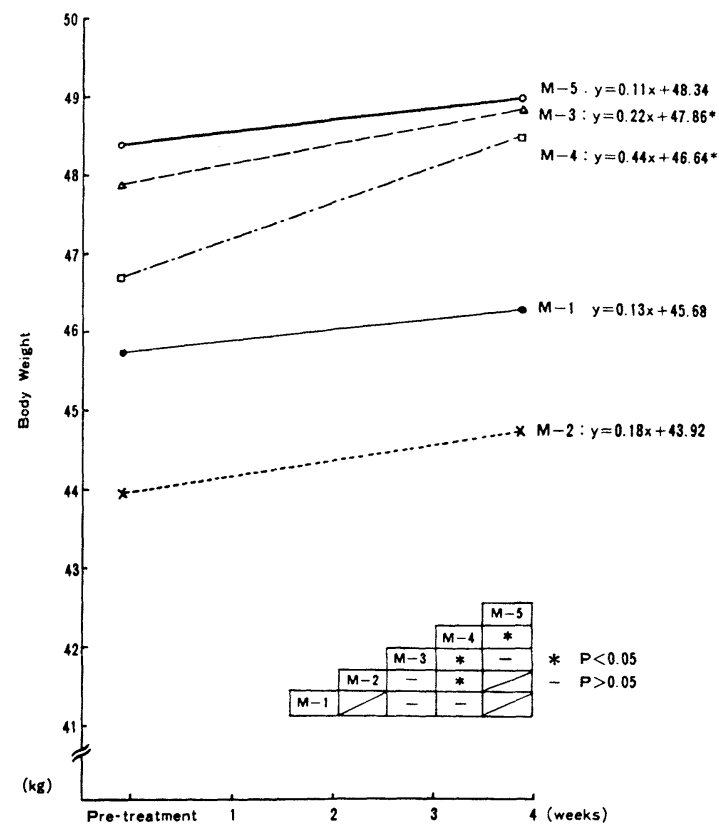

Fig. 8. Linear regression of body weight.
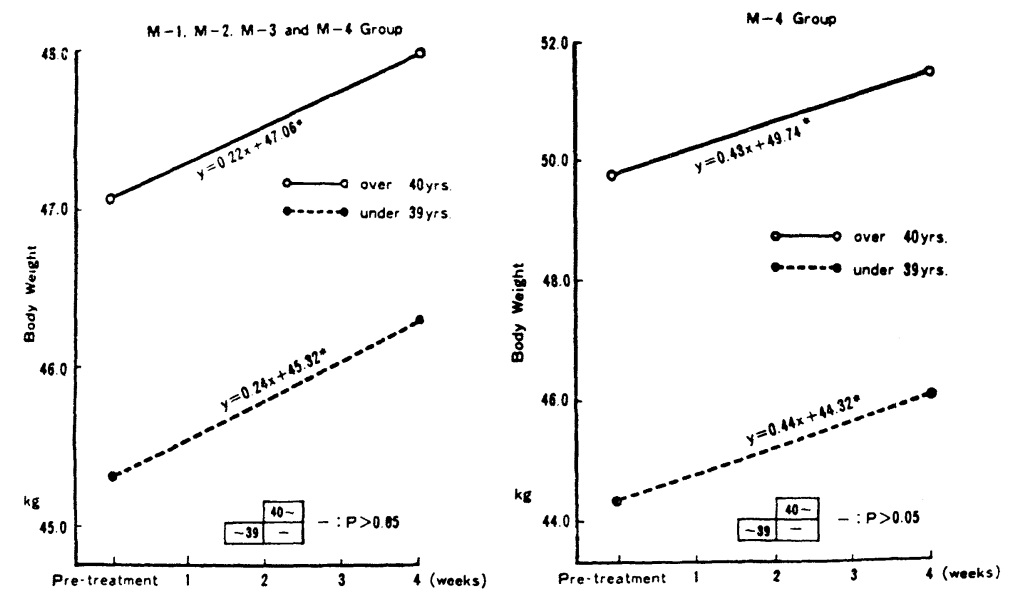

Fig. 9. Linear regression of body weight, stratified according to age.
(Geschickter 1945; Amaaki 1955) and androgenic hormone (Turpault 1937: Desmaret 1938; Loeser 1938; Atkins 1940; Konjetzny 1943; Fujimori 1952; Masuda 1955; Amaaki 1955; Tokuyama 1955; Yamamoto 1955). However, in order to avoid virilizing effects derivatives of testosterone, such as anabolic steroids are now being used.

Current androgen treatment for mastopathy is fairly inconsistent with regard to level of dosage, schedule of administration and duration of treatment. Therefore the authors attempted the present study to establish a rational program of drug administration. In these studies, greatest importance was attached to determination of variation in tumor size for objective evaluation of the drug effect. Reports in the literature thus for have described the drug effect in terms of diminution or disappearance of the tumor. Owing to the difference among the reports in the criteria of evaluation of drug effect, the difference in the drugs used, and the difference in dosage employed, there is some variation in the reported values. For instance, the rate of diminution in tumor size was reported as $52 \%$ by Amaaki with testosterone propionate and as $63 \%$ by the same author with 19nortestosterone-furan-propionate; as $90 \%$ by Saito with 19-nortestosterone propionate, and as $54.9 \%$ by Masuda with 2-methyl-dihydro- 
testosterone. In the author's previous studies using 19-nortestosterone-furan-propionate, complete disappearance of tumor was found in only $6.5 \%$ and diminution in $38.7 \%$ of the cases. The present results are not directly comparable with the above, since the current studies were based on planimetric determinations of the tumor area.

The primary aim was to see how different doses of the drug produced different effects. No particular difference among the groups was observed with regard to spontaneous pain, tenderness, and softening of the tumor. In contrast, an interesting result was obtained in tumor size: the higher the dose of drug, the higher was the diminishing effect of the tumor. Furthermore, a correlation between dose and weight gain was observed.

Stratified analysis of the data indicates a higher effectiveness of the drug in the younger age group. Effect of the drug was more profound in tumors larger in size or indefinitely bordered than in smaller tumors or those which were definitely bordered. However, age difference had no apparent effect on weight gain.

These data seem to suggest the types of mastopathy which should respond to androgen therapy. Further the effect of the present drug seems to be rather specific for mastopathy and differs from systemic anabolic action. The principles of hormonal therapy for mastopathy as deduced from the above reported findings may be stated as follows. Administration in somewhat high doses for 4 weeks; if response is sufficient treatment is halted; if the response is insufficient the drug is continued for 2 to 4 weeks more in a reduced dose; and if no response is observed surgical management is suggested.

\section{SUMMARY}

The present studies were undertaken in order to evaluate the therapeutic effectiveness and the side effects of 2-alpha-methyldihidrotestosterone propionate in patients with mastopathy, in view of the dose-response relationship.

Patients were divided into four groups according to dosage level of the test drug, plus a control group administered a depot type testosterone. Randomization of the patients was achieved by use of a table of random numbers. The drug was administered in double-blind fashion.

The treatments produced no significant difference among the groups with regard to spontaneous pain, tenderness, and softening of the tumor. However, regarding size, the tumor diminution was proportionate to the level of the dose. A similar tendency was observed in weight gain. Stratified analysis revealed that the tumor diminishing effect was higher in the younger age group, in tumors of larger size, and also in tumors bordered indefinitely. However, weight gain was not affected by age difference.

\section{ACKNOWLEDGEMENTS}

The authors wish to thank Shionogi Co., Ltd. for their kindness in preparing the 2alpha-methyl-dihydrotestosterone propionate used in this study.

\section{REFERENCES}

Amaaki, T. (1955). Jap. J. Surg. Soc. 56, 1034. (In Japanese)

Amaaki, T. (1965). Demeron Bunken Shu (Mochida Pharm. Co.) 5. (In Japanese)

Atkins, H.J.B. (1940). Lancet 1, 411.

Blackburn, C.M. (1959). Proc. Staff Meetings Mayo Clinic 34, 113.

Desmaret. (1938). Presse med. 11, 185.

Fujimori, M. (1952). cited from Fujimori (1962) Surg. Diagn. and Treatm. 4, 1.

Fujimori, M. (1957). Endocrinol. Japon. 4, 1. Fujimori, M. and M. Izuo (1962). Surg. Diagn. and Treatm. 4, 1. (In Japanese)

Fujimori, M. and M. Izuo (1963). Clin. Endocrinol. 11, 417. (In Japanese)

Geschickter, C.F. Diseases of the Breast. Lippincott. (1945).

Huggins, C. (1965). J. Exptl. Med. 104, 525. 
Izuo, M. (1965). Demeron Bunken Shu (Mochida Pharm. Co.) 7. (In Japanese)

Konjetzny, G.E. (1943). Zbl. Chir. Nr. 7.

Loeser, A.A. (1938). Lancet 1, 373.

Masuda, K. (1955). Shinryo 8, 55. (In Japanese)

Masuda, K. (1965). Sogo Rinsho 14, 684. (In Japanese)
Saito, S. (1961). Clin. Endocrinol. 9, 268. (In Japanese)

Tokuyama, E. (1955). Gan no Rinsho 1, 367. (In Japanese)

Turpault. (1937). J. des Prat.

Yamamoto, T. (1955). J. Nagoya City Med. Coll. 6, 171. (In Japanese) 